\title{
UTILIZAÇÃO DA TAXONOMIA II DA NANDA PARA AVALIAÇÃO DA ANSIEDADE PUERPERAL NA COMUNIDADEa
}

\author{
F laviana VIEI RA ${ }^{\mathrm{b}}, \mathrm{M}$ aria M árcia BACH I ON ${ }^{c}$, A manda Santos Fernandes COE L H Od, \\ A na Cláudia A ndrade CORDE IROe, A na Karina M arques SA LGE ${ }^{f}$
}

\section{RESUM 0}

Este trabalho tem como objetivo analisar a ocorrência do diagnóstico de enfermagem ansiedade, em puérperas no período imediato e tardio, no contexto da comunidade. T rata-se de um estudo descritivo transversal, de abordagem quantitativa. A amostra, não probabilística, foi constituída por 40 puérperas. $\mathrm{Na}$ coleta de dados foram utilizados procedimentos de entrevista, exame físico e observação no domicílio da puér pera. A ansiedade esteve presente em $80 \%$ das puér peras. Os fatores relacionados à ansiedade predominantes nas puérperas desta pesquisa foram o estresse (62,5\%) e a crise maturacional $(34,4 \%)$; evidenciados, principalmente, pela car acterística definidora comportamental: preocupações expressas em razão de mudanças em eventos da vida (46,9\%) e insônia (43,8\%). A lém de preocupações com o bem estar da criança e maior exigência com o aumento da carga de atividades. A aplicação dos diagnósticos de enfermagem favorece a identificação de prioridades de atendimento da assistência, e, per mite que medidas de inter venção precoce sejam adequadamente planejadas.

D escritores: Período pós-parto. Ansiedade. Diagnóstico de enfermagem.

\section{RESUMEN}

E ste trabajo tiene como objetivo analizar la ocur rencia de diagnóstico de enfer mería de ansiedad en puérperas en el periodo inmediato y tardío, en el contexto de la comunidad. Setrata de un estudio descriptivo transversal, deabordajecuantitativo. L a muestra no probabilística fue constituida por 40 puér peras. E n la recolección de datos fuer on utilizados procedimientos como entrevista, examen físico y observación en el domicilio de la puérpera. En $80 \%$ de ellas la ansiedad estuvo presente. L os factores predominantes fuer on el estrés $(62,5 \%$ y y la crisis demaduración $(34,4)$; evidenciados, princi pal mente, por la característica definidora comportamental: preocupaciones expresas en raz ón de cambios en momentos dela vida (46,9\%) einsomnio (43,8\%). A demás del bienestar del niño y la preocupación con el aumento de actividades. $L$ a aplicación de los diagnósticos deenfermería favorece la identificación de prioridades de atendimiento y per mite que medidas de inter vención precoz sean planificadas.

D escriptores: P eríodo de posparto. A nsiedad. D iagnóstico de enfer mería.

Título: U tilización de la Taxonomía II dela N AN DA para evaluación dela ansiedad puerperal en la comunidad.

\section{ABST RACT}

This paper aims to anal yz e the occur rence of nursing diagnosis A nxiety, in puerperal women immediately after and in the later period, within a community context. This is a transversal descriptive quantitative study. The no probabilistic samples consisted of 40 puerperal women. D uring data collection, interview procedures were used, in addition to physical examination and home observation. Anxiety was present in $80 \%$ of puerperal women. The anxiety-related factors predominating in puerperal women werestress (62.5\%) and maturational crisis (34.4\%); mainly evidenced by the behavioral defining characteristic: wor ries expressed due to changes in life events (46.9\%), and insomnia (43.8\%). In addition to concerns about the child's w ellbeing and an increased demands in burden of activities. The application of nursing diagnoses provides theidentification of priorities in the assistance and enables the adequate implementation of early intervention can be planned.

D escriptors: P ostpartum period. A nxiety. N ursing diagnosis.

T itle: The use of NAN DA's Taxonomy II for assessing puerperal anxiety within a community.

\footnotetext{
a E ste trabal ho deriva da disser tação de M estrado da primeira autora, desenvolvida em 2008 no Prog rama de Pós-G raduação em E nfer magem da Faculdade de Enfermagem da U niversidade Federal de G oiás (FE N / U F G).

${ }^{b}$ Enfer meira O bstetra, D outoranda do Programa de Pós-G raduação em E nfermagem da F E N / U F G, Professora da F E N / U F G, Pesquisadora do Grupo de Estudo em Saúde da M ulher, do Adolescente e da Criança (GESM AC), M embro do Núcleo de Estudos e Pesquisas em T ecnologias de Avaliação, Diagnóstico e Intervenção de Enfer magem e Saúde (N UTADIES), G oiânia, G oiás, Brasil.

c D outora Enfermagem, Professora T itular da FEN/ U F G, Coordenadora do N UTADIES, G oiânia, Goiás, Brasil.

¿ Enfermeira graduada pela FEN/U F G, G oiânia, G oiás, Brasil.

e Graduanda em Enfermagem do 10o período da FE N / UF G, G oiânia, Goiás, Brasil.

f D outora em Patologia, Professora A djunta da FEN/ U F G, Coordenadora do GESM AC, G oiânia, G oiás, Brasil.
} 


\section{INT RODUÇÃO}

0 sentimento de ansiedade é causado por situações estressoras que representam possibilidade de risco ou perigo, e, tem suas raízes nas reações de defesa, assim como o medo e o estresse. A ansiedade é acompanhada por modificações somáticas, diante de qual quer estressor que, na imaginação, represente ameaça(1).

A avaliação das condições emocionais e mentais do paciente é de extrema importância na prática da Enfermagem na detecção de algum nível de ansiedade visto que inúmeras condições emocionais são somatizadas repercutindo no aparelho cardiorrespiratório, gástrico ou neurológico(2), e no caso das puérperas interferindo na sua recuperação e ligação afetiva com a criança.

Diante de um estressor, respostas comportamentais básicas como "enfrentamento (ataque), evitação (fuga), passividade (colapso)" podem ser desenvolvidas. A resposta vai depender da magnitude e frequência do evento de vida estressor, assim como, dos fatores ambientais e genéticos, e, caso gere ativação fisiológica intensa pode haver um esgotamento dos recursos do indivíduo e assim, o surgimento de transtornos de ansiedade ${ }^{(1)}$.

Além da distinção entre ansiedade normal e patológica é válida a diferenciação entre ansiedade-estado e traço. A ansiedade-estado é a condição emocional transitória ou situacional, caracterizada por estado subjetivo de tensão, associado ao aumento da atividade do sistema nervoso autônomo. Já, a ansiedade-traço é a tendência para ansiedade ou ansiedade em geral, refere-se a diferenças individuais. Reflete característica relativamente estável da pessoa, que reage com ansiedade, diante de situações consideradas ameaçadoras ou estressantes ${ }^{(3)}$.

$\mathrm{NaT}$ axonomia II da N orth American $\mathrm{N}$ ursing Diagnosis Association - Internacional (NANDA-I), que representa uma das iniciativas para a criação de um sistema de linguagem partilhada pelos enfermeiros, é incluída entre seus 201 títulos diagnósticos a ansiedade, conceituada como: "Vago e incômodo sentimento de desconforto ou temor, acompanhado por resposta autonômica (a fonte é frequentemente não específica ou desconhecida para o indivíduo); sentimento de apreensão causado pela antecipação de perigo. É um sinal de alerta que chama a atenção para um perigo iminente e permite ao indivíduo tomar medidas para lidar com a ameaça(4)."

No ciclo gravídico-puerperal tudo é de caráter processual. Durante a gravidez, além das alterações físicas, como o desenvolvimento fetal, ocorre simultaneamente a aceitação do papel de mãe, que continua no puerpério, onde, as mudanças físicas, psicológicas e sociais são mais intensas e favorecem a vulnerabilidade emocional ${ }^{(5,6)}$.

No puerpério a mulher se ajusta a uma nova identidade - a identidade materna; aprende a lidar com o papel - de mulher-mãe; adapta-se ao novo el emento na família com identidade própria - o fiIho, e reestrutura as relações familiares e sociais $s^{(5)}$.

Neste sentido, a visão do profissional deve transpor as alterações fisiológicas, indo ao encontro ou aprofundando o reconhecimento das modificações psíquica e social nesta fase de construção de uma nova identidade e novo papel.

A Enfermagem pode contribuir significativamente com seus conhecimentos neste período que a mulher vivencia. Com o levantamento dos dados, identificação das respostas aos problemas de forma individualizada, considerando o contexto em que a puérpera está inserida, é fundamental para uma assistência planejada na fase puerperal( ${ }^{(7)}$.

Nos estudos encontrados, no Brasil, sobre o ciclo gravídico-puerperal são poucos aqueles direcionados ao diagnóstico de enfermagem ${ }^{(7-10)}$. 0 foco das pesquisas à saúde mental no ciclo gravídico-puerperal está voltado para a depressão pósparto e os estudos sobre ansiedade se concentram na gestação, desta forma a ansiedade é pouco estudada. Portanto, tornam-se necessárias pesquisas nesta vertente, pois, diagnósticos precisos são indispensáveis para conduzir as intervenções de enfermagem ${ }^{(11)}$.

A aplicação do processo de julgamento clínico dos diagnósticos de enfermagem no período pósparto, com vistas a afirmações diagnósticas baseadas em uma terminologia especial, ou seja, numa taxonomia diagnóstica, pode representar as respostas das puérperas e padronizar a linguagem da Enfermagem neste processo ou fase na vida da muIher.

D esta forma, propôs-se este trabal ho, tem como objetivo analisar a ocorrência do diagnóstico de enfermagem ansiedade, em puérperas no período imediato e tardio, no contexto da comunidade. 


\section{MET OD OLOGIA}

Trata-se de um estudo descritivo transversal, de abordagem quantitativa, realizado no Distrito Sanitário L este de G oiânia, G oiás, onde atuam as equipes de Estratégia de Saúde da Família (ESF). A pesquisa foi aprovada pelo Comitê de Ética em Pesquisa M édica Humana e A nimal do Hospital das Clínicas da U niversidade F ederal de G oiás (protocolo 185/07).

A amostra não probabilística, por conveniência, foi constituída por 40 puérperas. Foram feitos contatos com as equipes de ESF, entre janeiro e maio de 2008, para solicitação da relação das puérperas que poderiam atender os critérios de inclusão, após este levantamento as visitas domiciliárias foram previamente agendadas e acompanhadas pelo agente comunitário de saúde (ACS).

Os critérios de inclusão adotados foram: estar no puerpério imediato (até dez dias após o parto) ou tardio (do $11^{\circ}$ ao 45 o dia) (12), com criança viva, ter 18 anos completos ou mais, residir na área de cobertura da equipe de ESF da região estuda$\mathrm{da}$, independente da escolaridade, da renda, do estado civil, do tipo de parto e da paridade. Foram excluídas as puérperas que apresentassem diagnóstico médico de transtor no cognitivo ou psiquiátrico, que comprometesse a capacidade de autodeterminação.

0 instrumento de coleta de dados foi elaborado a partir dos fatores relacionados e características definidoras do diagnóstico estudado, segundo a T axonomia II NANDA-I, versão 2007-2008(13), que inclui 26 fatores relacionados (fatores causais ou coadjuvantes) para ansiedade (abuso de substância; ameaça e mudança na(o): função de papel, situação econômica, ambiente, estado de saúde, status do papel, padrões de interação; ameaça no autoconceito; ameaça de morte; associação familiar; conflito inconsciente quanto a metas de vida; conflito inconsciente quanto a valores essenciais da vida; contágio interpessoal; crise maturacional; crise situacional; estresse; exposição a toxinas; hereditariedade; transmissão interpessoal e necessidades não satisfeitas). N esta taxonomia, as características definidoras (indicadores, sinais e sintomas) deste diagnóstico incluem evidências comportamentais, afetivas, parassimpáticas, cognitivas e fisiológicas, totalizando 75 indicadores.

Para avaliação da presença dos fatores relacionados das características definidoras, realizaram- se buscas na literatura acerca dos parâmetros de avaliação de cada item e consultoria de três profissionais experientes da área, duas com experiência em obstetrícia e uma em diagnóstico de enfermagem, chegando a um roteiro final, que reuniu um conjunto de indicadores, para cada item, avaliados mediante procedimentos de entrevista, exame físico e observação participante, no domicílio da puérpera.

M ediante indicação dos ACS, as puérperas foram convidadas a participar, tendo sido esclarecidas quanto aos objetivos e procedimentos envolvidos no estudo e, aquelas que concordaram, manifestaram 0 aceite por escrito, mediante assinatura do T ermo de Consentimento L ivree E sclarecido. A coleta de dados transcorreu entre os meses de fevereiro e maio de 2008, por meio de um encontro com duração de duas horas. N este período, a puérpera esteve livre para prestar cuidados à criança.

Durante a coleta de dados foi preenchido 0 roteiro de coleta de dados ${ }^{(10)}$, e realizado o raciocínio diagnóstico, realizado em três etapas: análise dos indicadores clínicos das características definidoras e decisão sobre a presença das mesmas; análise dos indicadores dos fatores relacionados e decisão sobre a presença dos mesmos; análise das características definidoras encontradas e fatores relacionados, e decisão clínica que culminou com 0 julgamento da presença ou não do diagnóstico estudado.

Este julgamento foi posteriormente revisado por duas doutoras em enfermagem, sendo uma expert em diagnóstico de enfer magem e outra em enfermagem obstétrica. A dotou-sea T axonomialI da NA N DA-I (2007-2008)(13) para afirmação diagnóstica. Embora a taxonomia da NANDA tenha sido atualizada posteriormente( ${ }^{(4)}, 0$ diagnóstico Ansiedade não sofreu modificações.

A análise de dados foi feita mediante procedimentos de estatística descritiva (frequências simples e percentual).

\section{RESULT ADOS E DISCUSSÃO}

Foi identificada ansiedade em 32 (80\%) participantes. No grupo de puérperas estudado ( $\mathrm{N}=$ 40), a idade variou entre 18 e 35 anos, predominando a faixa etária de 21 a 25 anos (37,5\%). E m relação à escolaridade preponderou 0 ensino fundamental incompleto $(47,5 \%)$ e ensino médio com- 
pleto $(35,0 \%)$, a raça autorreferida predominante foi a parda $(65,0 \%)$. A maior parte estava fora do mercado de trabalho formal, sendo $55,0 \%$ do lar, e, $22,5 \%$ autônomas, as quais estavam envolvidas com atividades de trabal ho informal.

E $m$ relação à atividade laboral, $59,4 \%$ estavam envolvidas com atividades do lar e $40,6 \%$ tinham outras atividades remuneradas. Q uanto ao estado civil $82,5 \%$ encontravam-se casadas ou em união estável. A renda familiar predominante foi de 3 a 4 (42,5\%) salários mínimos. E m mais da metade dos casos, o número de residentes no domicílio foi de 3 a 4 (55,0\%), sendo constituído, geralmente, pela puérpera, o companheiro, um ou dois filhos.

As puérperas tiveram predominantemente parto cesáreo (65,0\%), de criança a termo $(90,0 \%)$; tinham até dois filhos (70,0\%), sendo que 37,5\% referiram gravidez planejada. A maioria buscou o pré-natal no primeiro trimestre de gestação $(80,0 \%)$, sendo que $62,5 \%$ fizeram no mínimo seis consultas.

Ao analisarem os sintomas de ansiedade em um grupo de gestantes, pesquisadores encontraram que as maiores prevalências de ansiedade traço estavam em mulheres com escolaridade entre um e oito anos (77,8\%), que vivem em união estável $(74,4 \%)$, com idade entre $18-24$ anos $(72,3 \%)$ e que não trabal havam (71,0\%). Observaram risco $35 \%$ maior para gestantes mais jovens, e, risco $40 \%$ maior para as de menor escolaridade ${ }^{(6)}$. Este diagnóstico pode continuar no pós-parto interferindo de negativamente na recuper ação da puérpera.

Além das modificações biológicas, neste período, ocorrem também as modificações e adaptações psicossociais que podem gerar ansiedade. Diversos fatores relacionados foram encontrados, confor me mostra a $T$ abela 1 , a seguir.

Os fatores relacionados à ansiedade predominantes nas puérperas desta pesquisa foram 0 estresse $(62,5 \%)$ e a crise maturacional $(34,4 \%)$.

As características definidoras mais observadas neste grupo foram: preocupações expressas em razão de mudanças em eventos da vida (46,9\%) e insônia (43,8\%). A s preocupações, que apresentam as dimensões de comportamentos e afeto, referemse a uma diversidade de temas, sendo os mais comuns em relação ao bem estar da criança e maior exigência com o aumento da carga de trabalho e das responsabilidades.
Estudo revela que no pós-natal a ansiedade está direcionada a preocupações e comportamentos de apego com a criança. A ansiedade possui implicações, pois foram encontradas correlações significativas entre os escores de ansiedade e de depressão mater na e o temperamento difícil das crianças $^{(14)}$.

As situações provocadoras de estresse podem ser agrupadas em acontecimentos vitais, acontecimentos diários menores e situações de tensão crônica. Os acontecimentos vitais são as mudanças importantes na vida, como por exemplo, o nascimento de um filho, e podem gerar resposta de estresse nos indivíduos a eles expostos ${ }^{(1)}$.

A percepção que a pessoa tem de uma determinada situação, influencia o nível de estresse experimentado, e, não apenas a situação causadora do estresse, ou seja, as circunstâncias não são unicamente responsáveis pelo o aumento do estresse, mas também a forma com que pessoa lida e responde a tais circunstâncias ${ }^{(15)}$.

A insônia, aliada às novas demandas, contribui para o surgimento de outras características definidoras: a irritabilidade (37,5\%), o nervosismo $(31,2 \%)$ e a dificuldade para concentra-se $(21,9 \%)$. Além disso, com o nascimento da criança houve mudanças na situação econômica de várias puérperas, que representou para estas, preocupações com o desemprego e diminuição da renda familiar, configurando nos fatores relacionados ameaça ou mudança na situação econômica $(28,1 \%)$.

Baseados no uso da escala de autoavaliação de ansiedade-traço e ansiedade-estado, pesquisadores encontraram a prevalência de 44,8\% para ansiedade-puerperal-estado, e $46,1 \%$ para ansiedade-puerperal-traço. Referiram que se tivessem usado critérios psiquiátricos, provavelmente teriam uma estimativa de transtorno de ansiedade bem menor ${ }^{(3)}$.

A valiados sintomas de angústia no pós-parto, como: a depressão, ansiedade e estresse, em uma pesquisa ${ }^{(16)}$, foi identificado que $10 \%$ das mulheres tiveram sintomas de ansiedade e estresse sem depressão, 13\% apresentaram sintomas de ansiedade em isolamento ou em combinação com depressão. Sendo que do total de mulheres, $25 \%$ tiveram possibilidade de apresentar depressão. Evidenciase a importância de não somente avaliar a depressão no pós-parto, mas também a ansiedade e 0 estresse como fatores psicológicos ${ }^{(16)}$. 
T abela 1 - Fatores relacionados e características definidoras de ansiedade $(n=32)$ em puérperas no período imediato e tardio. Distrito Sanitário Leste, G oiânia, G O, fev./ maio 2008.

\begin{tabular}{|c|c|c|}
\hline Fatores relacionados & $f$ & $\%$ \\
\hline $\begin{array}{l}\text { E stresse } \\
\text { Crise maturacional } \\
\text { N ecessidades não satisfeitas } \\
\text { A meaça ao autoconceito } \\
\text { M udança nos padrões de interação } \\
\text { Crise situacional } \\
\text { M udança na situação econômica } \\
\text { M udança na função de papel } \\
\text { A meaça na situação econômica } \\
\text { M udança no ambiente } \\
\text { A meaça nos padrões de interação } \\
\text { Conflito inconsciente quanto à meta de vida }\end{array}$ & $\begin{array}{l}20 \\
11 \\
09 \\
08 \\
08 \\
07 \\
06 \\
05 \\
03 \\
02 \\
01 \\
01\end{array}$ & $\begin{array}{r}62,5 \\
34,4 \\
28,1 \\
25,0 \\
25,0 \\
21,9 \\
18,8 \\
15,6 \\
9,3 \\
6,3 \\
3,1 \\
3,1\end{array}$ \\
\hline Características definidoras & $f$ & $\%$ \\
\hline $\begin{array}{l}\text { Comportamentais } \\
\text { Preocupações expressas em razão de mudanças em eventos da vida } \\
\text { Insônia } \\
\text { Produtividade diminuída } \\
\text { Inquietação } \\
\text { Observação atenta }\end{array}$ & $\begin{array}{l}15 \\
14 \\
06 \\
03 \\
02\end{array}$ & $\begin{array}{r}46,9 \\
43,8 \\
18,8 \\
9,3 \\
6,3\end{array}$ \\
\hline $\begin{array}{l}\text { A fetivas } \\
\text { Preocupada } \\
\text { I rritabilidade } \\
\text { N ervosa } \\
\text { Ansiosa } \\
\text { A preensiva } \\
\text { Angústia } \\
\text { Pesarosa } \\
\text { Desamparo aumentado e persistente }\end{array}$ & $\begin{array}{l}15 \\
12 \\
10 \\
06 \\
06 \\
04 \\
03 \\
02\end{array}$ & $\begin{array}{r}46,9 \\
37,5 \\
31,2 \\
18,8 \\
18,8 \\
12,5 \\
9,4 \\
6,3\end{array}$ \\
\hline $\begin{array}{l}\text { Cognitivas } \\
\text { Dificuldade para concentrar-se } \\
\text { Bloqueio de pensamento } \\
\text { E squecimento }\end{array}$ & $\begin{array}{l}07 \\
04 \\
01\end{array}$ & $\begin{array}{r}21,9 \\
12,5 \\
3,1\end{array}$ \\
\hline $\begin{array}{l}\text { Parassimpáticas } \\
\text { Fadiga } \\
\text { Fisiológicas }\end{array}$ & 04 & 12,5 \\
\hline $\begin{array}{l}\text { Aumento da tensão } \\
\text { Tensão facial }\end{array}$ & $\begin{array}{l}04 \\
02\end{array}$ & $\begin{array}{r}12,5 \\
6,3\end{array}$ \\
\hline $\begin{array}{l}\text { Simpáticas } \\
\text { A umento da pressão sanguínea } \\
\text { Vasoconstrição superficial }\end{array}$ & $\begin{array}{l}01 \\
01\end{array}$ & $\begin{array}{l}3,1 \\
3,1\end{array}$ \\
\hline
\end{tabular}

Esta situação de ansiedade é esperada para o período puerperal, uma vez que com a chegada da criança, tanto a mulher quanto sua família e seu meio social, passam por modificações e adaptações com este período de transição, e, mesmo para aquelas que já vivenciaram o puerpério anteriormente, esta fase será sempre uma experiência peculiar.

Por isso uma adequada intervenção durante o pré-natal com orientações concernentes ao pe- ríodo puerperal, como forma de antecipação relativa do evento, pode resultar em comportamentos de ajustes para enfrentamento do estresse que será vivenciado posteriormente.

0 fator relacionado - necessidade não satisfeita - foi expresso por $28,1 \%$ das puérperas, devido a desejos de ter uma condição de vida melhor que poderia garantir um futuro digno para sua criança, ter casa própria, melhorar imagem corporal, 
preocupações com os outros filhos, voltar a estudar para conseguir um emprego e desejo de amamentar eficazmente.

A necessidade de boas condições de vida foi citada em uma pesquisa, como a segunda maior necessidade das puérperas que tem como expressão "viver em sociedade; ter acesso a lazer, alimentação, moradia, transporte e viver em meio ambiente adequado". A primeira necessidade das puérperas questionadas foi a necessidade do exercício de cidadania, de serem respeitadas e suas necessidades e poder exercitar seu direitos sexuais. Evidencia-se, então, que as necessidades sociais em saúde, que extrapolam o nível de ação das equipes de saúde, interferem grandemente na saúde psíquica dessas mulheres, caracterizando um aumento dos níveis estressores e podendo também interferir nos níveis de ansiedade ${ }^{(17)}$.

No fator relacionado ameaça de autoconceito $(25 \%)$ verificou-se que as mudanças do corpo foram evidenciadas, como aumento de peso e da circunferência a abdominal, onde a aparência passara a não agradar mais estas mulheres, representando desconfor to e baixa autoestima.

$\mathrm{E} m$ relação à mudança no padrão de interação $(25,0 \%)$ foi devido a dificuldades relacionais entre a mulher e alguns familiares, principalmente com os outros filhos e com o pai da criança.

A puérpera vivencia diver sas facetas neste período, dentre elas o "ser mãe no puerpério". A vivência materna engloba diversos aspectos, tanto envolvendo o cuidado com a criança, sua relação conjugal, as diversas tarefas sociais e sua imagem pessoal feminina. $\mathrm{Na}$ nova identidade incluem $\mathrm{O}$ descuido com a imagem pessoal feminina e a renúncia ou desistência temporária dos divertimentos e da busca de um novo emprego, face à prioridade de atender a criança ${ }^{(18)}$.

A participação do pai no puerpério é fundamental para a boa interação da família, por meio do desenvolvimento de atitudes de cuidado com a companheira, dedicação e preocupação com a saúde da criança, reconhecendo que a companheira e o recém-nascido requer em mais dedicação/ tempo nesta fase $\mathrm{e}^{(19)}$.

A falta de apoio, o sentimento de solidão e a necessidade de pessoas para auxilio em suas atividades foram alguns dos motivos que contribuíram para a crise situacional (25\%) entre as puérperas.

0 fator relacionado - mudança de função de papéis - presente em $15,6 \%$ das puérperas, justifi- ca-se pelo fato delas se encontrarem em uma fase de transição, de readaptação da sua vida e da família, em maturação de uma nova identidade: a de ser mãe; adicionadas a novas responsabilidades, atribuições e afazeres; incapacidade de fazer as coisas como antes; mudanças de hábitos devido à presença da criança recém-chegada.

0 nascimento da criança faz com que a puérpera tenha receio em não se adaptar à mater nidade, ou não ser capaz de atender todas as necessidades que a criança traz consigo.

A ansiedade por sua vez, pode interferir em outras respostas, especialmente, naquelas relativas à amamentação, fator relatado por algumas puérperas com preocupação, ou seja, causador de estresse e conseqüentemente 0 aumento da ansiedade. A resolução de dificuldades, no contexto de ansiedade, pela própria puérpera pode não ser efetiva, necessitando de apoio dos profissionais de saúde ${ }^{(10)}$.

$\mathrm{Na}$ avaliação da ansiedade em nutrizes verificou-se que, tanto as puérperas hipogalactas quanto as normogalactas, apresentaram grau médio de ansiedade; em que as nutrizes com traço de maior nível de ansiedade são as multíparas hipogalactas (39,9 pontos da escala A-estado) seguido pelas primíparas normogalactas ( 36,6 pontos da escala A-estado), todas no 100 dia pós-parto. No 30 dia de pós-parto, a média de ansiedade das nutrizes foi inferior, período onde possivelmente as maiores dificuldades foram solucionadas além de uma melhor adaptação(20).

A caracterização da ansiedade materna durante o pós-parto tem grande importância, visto que pode influenciar grandemente na produção e ejeção láctea principalmente em decorrência do comportamento choroso do recém-nascido e preocupação como o peso deste, que por sua vez aumentam ainda mais os níveis de ansiedade ${ }^{(20)}$.

E mbora as puérperas apresentem desejo em amamentar, alguns fatores influenciam para que tal aconteça de forma ineficaz, como: a ansiedade materna. A ansiedade é um fator relacionado do diagnóstico de enfermagem amamentação ineficaz, evidenciado em puérperas ${ }^{(10)}$.

A não efetividade da amamentação pode ser considerada um grande estressor para determinadas mulheres, trazendo insegurança e aumentando os níveis de ansiedade. A lém disso, tanto o comportamento da criança pode exercer grande influência no estado de ansiedade materna como mães 
ansiosas podem transmitir ansiedade para as crianças que ficam mais agitadas, ocorrendo assim um círculo vicioso ${ }^{(20)}$.

Assim, ao se examinar uma mulher no puerpério, deve-se inicialmente realizar uma avaliação do seu estado psíquico, pois é comum que a muIher experimente sentimentos contraditórios e sinta-se insegura neste momento da vida.

\section{CONCLUSÕES}

A análise dos diagnósticos de enfermagem no puerpério favorece a identificação de prioridades de atendimento da assistência, os conteúdos essenciais a serem abordados em processos educativos, e direciona 0 avanço nas áreas de pesquisa de inter venções de enfermagem.

Com esta pesquisa pode-se observar que no período puerperal o diagnóstico de enfermagem ansiedade surge devido a grandes preocupações, como a capacidade de cuidar do recém-nascido e ainda dar continuidade às atividades realizadas antes da gravidez. A avaliação desta resposta humana foi efetiva devido à proximidade dos pesquisadores com a realidade da puérpera.

A ansiedade precisa ser investigada no período antenatal para que seja assistida antes de se instalar no puerpério, diminuindo assim um dos fatores de risco para depressão. Ainda, ser avaliada 0 mais próximo do contexto em que a puérpera está inserida, como a assistência no domicílio.

A identificação dos fatores relacionados e das características definidoras da ansiedade materna permite que medidas de intervenção precoce sejam adequadamente planejadas e implementadas, e que, possíveis os efeitos da ansiedade materna em outras respostas humanas, especialmente, naquelas relativas à amamentação, possam ser reduzidos.

Com isso é essencial o trabalho do enfer meiro enquanto profissional que oferece apoio e vínculo, através da escuta dos anseios da puér pera, para o fortalecimento e autonomia diante das novidades e mudanças vividas nesta fase de vida.

Estudos abrangentes da associação da ansiedade no pré e pós-natal são necessários para que a ansiedade não seja um agravante tanto para readaptação fisiológica materna quanto para a interação entre mãe e filho. N ão obstante, é fundamental a distinção das diversas preocupações da gestante/ puérpera dos transtornos ansiosos, como, a preocupação com imagem corporal e saúde do recém-nascido o que não significa que estas mães apresentem quadros psiquiátricos típicos, mas são frequentes no puerpério.

A partir deste trabalho, com o conhecimento dos fatores associados à ansiedade, novas pesquisas podem ser realizadas com intervenções por meio da implantação de programas preventivos na atenção primária, principalmente, sob a responsabilidade do profissional de enfermagem que tanto atua na assistência ao ciclo gravídico puer peral.

\section{RE FE RÊ NCIAS}

$1 \mathrm{M}$ argis R, Picon P, Cosner AF, Silveira RO. Relação entre estressores, estresse e ansiedade. Rev Psiquiatr Rio Gd Sul. 2003;25 Supl 1:65-74.

2 Barros ALBL. Anamnese e exame físico: avaliação diagnóstica de enfermagem no adulto. Porto A legre: Artmed; 2002.

3 Faisal-Cury A, M enezes PR. Ansiedade no puerpério: prevalência e fatores de risco. Rev Bras Ginecol Obstet. 2006;28(3):171-8.

$4 \mathrm{~N}$ orth A merican Nursing Diagnosis Association Inter national. D iagnósticos de E nfermagem da NA N DA: definições e classificação 2009/ 2011. Porto Alegre: Artmed; 2010.

$5 \mathrm{~N}$ avajas $\mathrm{F}$ ilho $\mathrm{E}$. Os processos de identificação e introjeção na gravidez. In: Zugaib $M$, T edesco JJA, Quayle J. Obstetrícia psicossomática. São Paulo: Atheneu; 1997. p. 10-4.

6 A raújo D M R, Pacheco AHR N, Pimenta A M , Kac G. Prevalência e fatores associados a sintomas de ansiedade em uma coorte de gestantes atendidas em um centro de saúde do município do Rio de Janeiro. Rev Bras Saúde M ater Infant. 2008;8(3):333-40.

7 Pereira SVM , Bachion M M. Diagnósticos de enfermagem identificados em gestantes durante o prénatal. Rev Bras Enferm. 2005;58(6):659-64.

8 Abrão ACF V, Guitiérrez M GR, M arin HF. Diagnóstico de enfermagem amamentação ineficaz: estudo de identificação e validação clínica. A cta Paul E nferm. 2005;18(1):46-55.

9 Arcanjo Z, Alves VM, Palmeira ILT, Lopes M VO. Diagnósticos de enfermagem referentes ao sono e repouso de gestantes. Rev Enferm UERJ. 2006;14(3): 378-84. 
10 Vieira F. Diagnósticos de enfer magem identificados em puérperas no período imediato e tardio no contexto da comunidade [ disser tação] . G oiânia: F aculdade de Enfer magem, U niversidade F ederal de G oiás; 2008.

11 L unney M . Critical thinking and accuracy of nurses' diagnoses: part I: risk of low accuracy diagnoses and new views of critical thinking. Rev Esc Enferm U SP. 2003;37(2):17-24.

12 Rezende J. Obstetrícia. 10aㅡ ed. Rio de Janeiro: Guanabara Koogan; 2005.

13 N orth American Nursing Diagnosis Association. D iagnósticos de enfermagem da NAN D A: definições e classificação 2007/ 2008. Porto Alegre: Artmed; 2008.

14 Correia LL, L inhares M BM . A nsiedade mater na nos períodos pré e pós-natal: revisão da literatura. Rev Latino-A m Enfermagem. 2007;15(4):677-83.

14 Calderero ARL, M iasso Al, Corradi-W ebster CM . Estresse e estratégias de enfrentamento em uma equipe de enfer magem de Pronto A tendimento. Rev
Eletrônica Enferm [I nternet] . 2008 [ citado 2010 fev 15];10(1):51-62. Disponível em: http:/ / www.fen. ufg.br/ revista/ v10/ n1/ v10n1a05.htm.

16 M iller RL, Pallant JF, Negri LM . Anxiety and stress in the postpartum: is there more to postnatal distress than depression? BM C Psychiatry. 2006;6(12):111.

17 Souza KV, Cubas M R, Arruda DF, Carvalho PRQ, Carvalho CM G. A consulta puer peral: demandas de mul heres na perspectiva das necessidades sociais em saúde. Rev G aúcha Enferm. 2008;29(2):175-81.

18 Saraiva ERA, Coutinho, M PL. 0 sofrimento psíquico no puerpério: um estudo psicossociológico. Rev M al-E star Subj. 2008;8(2):505-27.

19 Oliveira EM F, Brito RS. A ções de cuidado do pai no puerpério. Esc Anna N ery Rev Enferm. 2009;13(3): 595-601.

20 A ragaki I M M , Silva IA, Santos LF. T raço e estado de ansiedade de nutrizes com indicadores de hipogalactia e nutrizes com galactia normal. Rev Esc Enferm USP. 2006;40(3):396-403.
Endereço da autora / Dirección del autor / Author's address:

Flaviana Vieira

Rua 227, Qd. 68, s/ n, Setor Leste U niversitário

74605-080, G oiânia, G 0

E-mail: flavianamori@gmail.com
Recebido em: 31/ 05/ 2010

A provado em: 28/ 09/ 2010 